\title{
CALCULATED GAMMA RAY RESPONSE CHARACTERISTICS OF SEMICONDUCTOR DETECTORS
}

\author{
K. M. WAINIO* and G. F. KNOLL \\ Department of Nuclear Engineering, The University of Michigan, Ann Arbor, Michigan
}

Received 5 March 1966

\begin{abstract}
A Monte Carlo computer program has been used to calculate characteristics of the response of fully depleted silicon and germanium radiation detectors to monoenergetic gamma rays. Data for total absorption probability, intrinsic efficiency, escape peak efficiency and pulse height spectra are presented as functions of detector thickness and photon energy. Other parameters of interest in analysing detector response are also given. The results
\end{abstract}

\section{Introduction}

The development of the ion-drift technique in semiconductor materials has made possible the fabrication of solid state radiation detectors with relatively large sensitive volume. Consequently, the gamma ray detection efficiency of these devices has been significantly improved and, because of their outstanding energy resolution, increasing attention is being given to their application in gamma ray spectroscopy. This paper presents the results of a computer program used to calculate some characteristics of the response of silicon and germanium detectors to monoenergetic gamma rays.

The objective of these calculations is to predict the total absorption probability, escape peak efficiency, peak to total ratio and pulse height distribution as a function of detector thickness and incident photon energy. Other parameters characteristic of some details of the gamma ray interaction are also extracted to provide a more complete description of the detection process.

\section{Description of the computer program}

The program is written for a detector configuration consisting of a right circular cylinder of prescribed radius and height. The detector material can be either pure silicon or germanium and crystalline effects are neglected. The depletion layer, or sensitive volume, is also assumed to be a right circular cylinder whose radius is the same as that of the whole detector. The surface of the detector exposed to the primary gamma ray flux is assumed to be coplanar with one surface of the depletion layer so the detector has no front surface dead layer. In general, the program provides for an

* Present Address: Bendix Systems Division, Ann Arbor, Michigan. of a second Monte Carlo calculation of electron migration in silicon and germanium are employed to account for the leakage of secondary electrons from the detector volume. Bremsstrahlung energy loss by electrons is also simulated. The calculations are expected to be applicable in those cases in which secondary electron energies do not exceed $2 \mathrm{MeV}$. Comparison with experiment shows good agreement within this limitation.

undepleted region of arbitrary thickness behind the sensitive volume. However in all the results reported here the detectors are assumed to be fully depleted. Throughout the calculations, it is assumed that the output pulse height from a given gamma ray interaction is proportional to the total electron energy deposited within the depletion layer.

Two distinct types of transport processes are considered in the program. First, gamma rays from the source, together with secondary gamma rays formed in primary interactions, are followed through the detector material using biased Monte Carlo sampling procedures. Second, at each interaction point the migration of secondary electron(s) formed through the photoelectric, Compton scattering, or pair production processes, is simulated using the results of a separate Monte Carlo program. Because typical secondary electron ranges are of the same order of magnitude as detector dimensions, the transport of these energetic electrons into or out of the depletion layer has an important effect on the detector response.

The cross-section data for the three primary gamma ray interaction processes of photoelectric absorption, Compton scattering and pair production were obtained from the tabulations of Storm et al. ${ }^{1}$ ). All photoelectrons are assumed to be produced with a kinetic energy equal to the incident photon energy minus the $\mathrm{K}$-shell binding energy. Their angular distribution with respect to the incoming photon direction is obtained by sampling the distribution developed by Sauter ${ }^{2}$ ) for the polar angle and by assuming azimuthal isotropy. All atomic excitation energy is considered to be locally absorbed. Compton scattering events are described by the Klein-Nishina differential cross section which is sampled using a rejection procedure developed by $\mathrm{Kahn}^{3}$ ). Each scattered photon is treated 
in the same fashion as primary gamma rays to determine its further fate. In pair production events, it is assumed that the kinetic energy shared by the electronpositron pair, $h v-2 m_{0} c^{2}$, is partitioned such that the energy given to either member of the pair follows a uniform distribution from zero to the full energy. The angular distributions of the electron pair are sampled from the relativistic approximation given by Heitler ${ }^{4}$ ). Two annihilation quanta of energy $m_{0} c^{2}$ are assumed to be emitted isotropically and diametrically opposed after the positron member of the pair has lost all its initial kinetic energy. To simplify the model, the annihilation radiation is assumed to be produced at the original pair production site. Further tracking of the annihilation photons again follows the procedure used for primary gamma rays.

The escape of secondary electrons from the sensitive volume of the detector is simulated using the results of a second Monte Carlo program, stored in tabular form in the computer memory. These results give the probability for electron escape from semi-infinite media of silicon and germanium. The data are incorporated into the gamma ray calculations by evaluating the probability of escape from the sensitive volume for each secondary eleciron formed in all gamma ray interactions. For these purposes, no distinction is made between positrons and electrons. In those cases in which a random sampling of the probability indicates that the electron has escaped, it is assumed to carry off the average escape energy appropriate to its initial conditions. It is sometimes possible for the electron to escape from more than one detector surface. In such a case the semifinite medium data is successively applied to each surface to calculate the net leakage probability.

The electron transport program ${ }^{5}$ ) is based on a Monte Carlo simulation of the electron path using a large number (100-200) of small path length intervals. Each of these path segments is assumed to be equivalent to a thin foil so that distribution functions developed for and verified by foil transmission experiments can be used to represent the energy loss and net angle of scatter in the path length interval. These distribution functions are sampled using Monte Carlo techniques and the entire trajectory of the electron is simulated by combining the results of the successive path segments. The program was applied to semi-infinite media of silicon and germanium with the result that both the escape probability and the average energy at escape may be well represented as functions of only the two variables $x / R$ and $\mu$, independent of the initial electron energy. Here $x$ represents the extrapolated distance to the boundary along the initial velocity vector, $R$ is the electron range and $\mu$ is the cosine of the angle made by the initial velocity with the plane of the boundary. These "universal" curves are good approximations to the calculated data for electron energies up to approximately $2 \mathrm{MeV}$ and are used in the gamma ray program to minimize the computer time necessary to sample the escape data. Numerical values for electron ranges in silicon and germanium have been taken from the tabulation of Nelms ${ }^{6}$ ).

Bremsstrahlung energy loss by secondary electrons is also taken into account in those cases in which the electron does not escape from the depletion layer. The data of Zerby and Moran ${ }^{7}$ ), which give the differential number spectra for the total radiation emitted by an electron stopped in $\mathrm{NaI}$, were applied to silicon and germanium by assuming that the bremsstrahlung crosssection varies as $Z^{2}$ and the rate of ionization energy loss varies as $Z$. The number and energy of bremsstrahlung photons are obtained by random sampling from this distribution and each photon is assumed to be emitted isotropically from the initial position of the electron. The photons are then permitted to escape with the probability $\exp (-\mu x)$, where $x$ is the distance the photon must travel to escape from the depletion layer and $\mu$ is the total absorption coefficient.

Several biasing arrangements are employed to increase the efficiency of the Monte Carlo process. Each source photon is forced to interact within the detector by one of the three primary interaction processes. These interactions may be preselected to be all of one type, or may be sampled from all three according to the relative magnitudes of the cross-sections. The former procedure is useful when investigating specific detector properties (for example, the contribution of primary Compton events to the full energy peak) while the latter procedure simulates the actual behavior of the detector. Secondary photons (Compton scattered gamma rays and annihilation radiation) are also forced to interact and the histories involved are assigned the appropriate statistical weight. In this way, statistically significant information can be obtained at a greater economy in computer time than with unbiased Monte Carlo simulation.

\section{Comparison with experimental data}

To test the accuracy of the calculations, comparisons were made between the computed and experimental data for the following detector response parameters:

1. the total absorption probability;

2. the double escape pair peak efficiency;

3. the peak to total ratio;

4. the pulse height distributions produced by ${ }^{60} \mathrm{Co}$ 


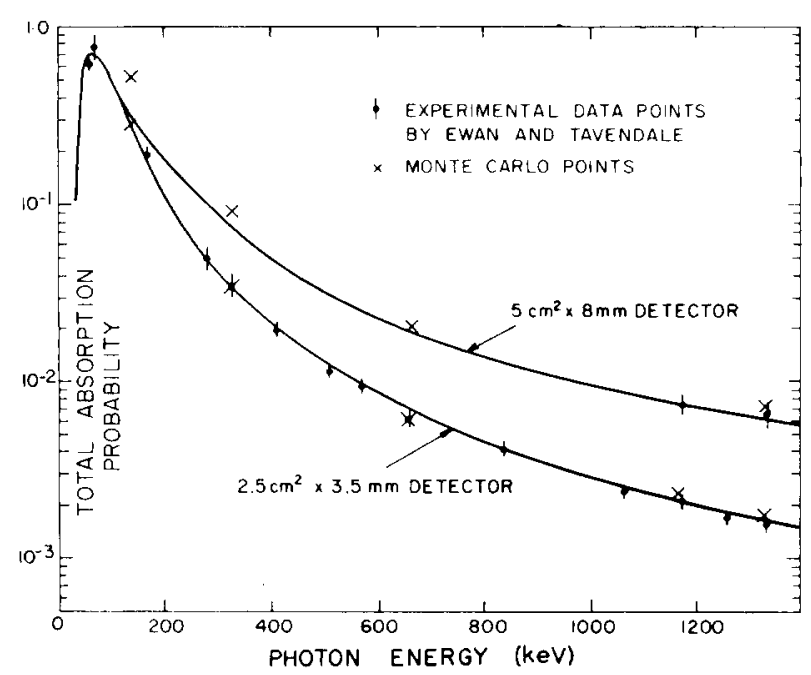

Fig. 1. Comparison of computed and experimental data for the total absorption probability in germanium detectors. Experimental data by Ewan and Tavendale ${ }^{8}$ ).

and $\mathrm{Th}\left(\mathrm{B}+\mathrm{C}+\mathrm{C}^{\prime \prime}\right)$ gamma rays. The experimental data shown is that of Ewan and Tavendale ${ }^{8}$ ), obtained using lithium-drifted germanium detectors.

The predicted total absorption probability, that is the probability that the incident photon interacts in the detector and the full source energy of the photon is totally absorbed, is compared with experimental values in fig. 1. The experimental data points with the indicated error flags and the curves drawn through the data points have been taken directly from ${ }^{8}$ ). Experimental data are shown for two detectors: one with a surface area of $2.5 \mathrm{~cm}^{2}$ and $3.5 \mathrm{~mm}$ thickness and the other with an area of $5 \mathrm{~cm}^{2}$ and $8 \mathrm{~mm}$ thickness. Monte Carlo data are shown for the same two detector thicknesses; however, in both cases the detector area is assumed to be $2.5 \mathrm{~cm}^{2}$. Error estimates are not indicated for the Monte Carlo data but in all cases the statistical error is less than 3 per cent.

The computed values for the $3.5 \mathrm{~mm}$ detector are in good agreement with the experimental data, but there is a systematic discrepancy in the case of the $8 \mathrm{~mm}$ detector. Furthermore, the calculations for the latter case having been made for a detector of half the actual surface area, we estimate the values would be about $10 \%$ greater for the larger surface area, making the discrepancy even greater. The experimental values, however, could be as much as $10 \%$ too low for the $8 \mathrm{~mm}$ detector due to uncertainties in the solid angle measurement ${ }^{8}$ ). Such a correction would bring the data into fair agreement, except for the values at $136 \mathrm{keV}$. Here the effect of a $400 \mu \mathrm{m}$ thick front surface dead layer, not taken into account in the calculation, is most important and

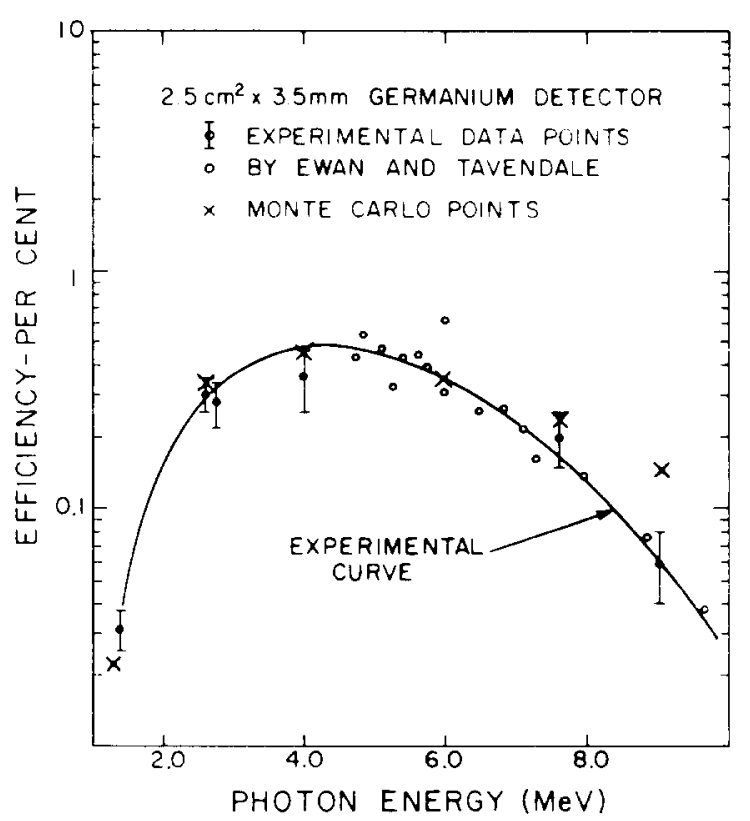

Fig. 2. Comparison of computed and experimental data for the double escape peak efficiency in a germanium detector. Experimental data by Ewan and Tavendale ${ }^{8}$ ).

would tend to make the computed values too high. The dead layer could also account for the systematic discrepancy at the higher energies since the effective thickness of the detector is less than the $8 \mathrm{~mm}$ thickness employed in the calculations.

For photon energies greater than $2 \mathrm{MeV}$, gamma ray spectroscopy is often based on the double escape peak. Fig. 2 presents a comparison of computed and experimental data for the double escape pair peak efficiency. The experimental data rrom $^{8}$ ) have been obtained with the same $3.5 \mathrm{~mm}$ thick detector used for the total peak efficiency data quoted above. The data points marked by the crosses are estimates of the double escape pair peak efficiency obtained with the Monte Carlo program. Except at very high energies, the predicted values are in good agreement with the experimental data. The discrepancy at high energy is most likely due to inaccurate description of the electron leakage probability. As indicated above, the data used to calculate the escape probability are not expected to be accurate for electron energies in excess of $2 \mathrm{MeV}$. Part of the discrepancy at the high energies may also be due to inflight annihilation of the positron or to an annihilation mode other than two quanta annihilation since such processes are not included in the Monte Carlo simulation.

Comparison of other detector response parameters do not provide as useful tests of the calculation because 


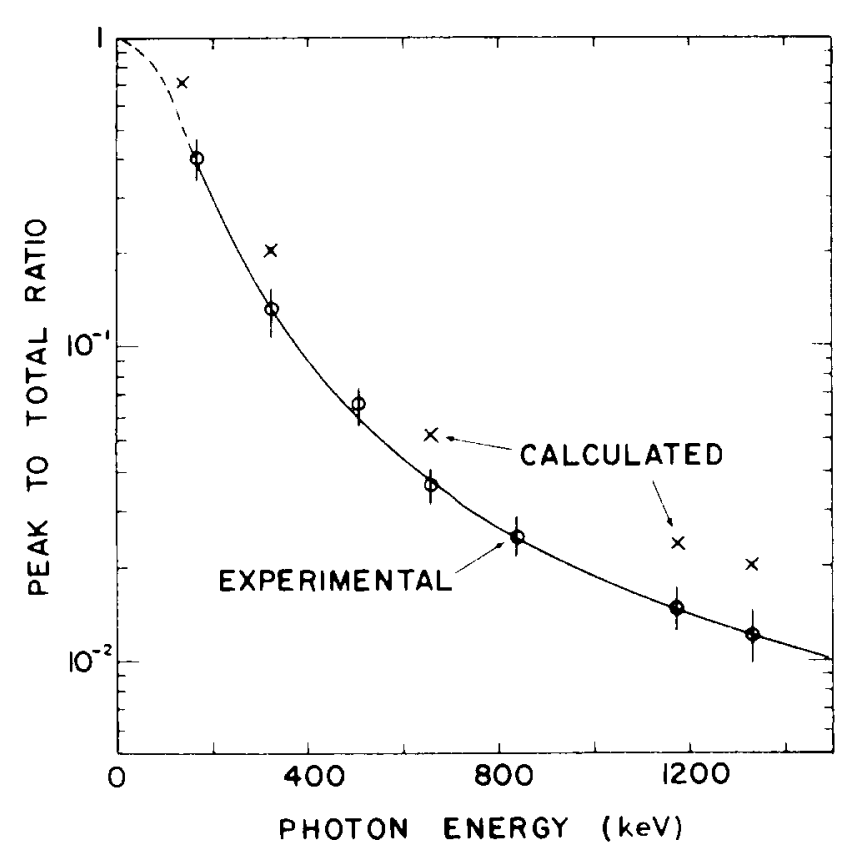

Fig. 3. Comparison of computed and experimental data for the peak to total ratio in a $3.5 \mathrm{~mm} \times 2.5 \mathrm{~cm}^{2}$ germanium detector. Experimental data by Ewan and Tavendale ${ }^{8}$ ).

of the presence of scattered radiation background in the experiment. For example, a comparison of the predicted and experimental data for the peak to total ratio obtained by Ewan and Tavendale ${ }^{8}$ ) using the $3.5 \mathrm{~mm}$ detector is shown in fig. 3. The computed values are considerably larger than the experimental data as expected since the experimental data for the total count include photons scattered from the source and detector mount. The discrepancy between the computed and experimental results increases with increasing source energy, probably because of the relative increase in the probability for Compton scattering.

A comparison of the predicted and observed pulse height distribution for ${ }^{60} \mathrm{Co}$ gamma rays incident on the same $3.5 \mathrm{~mm}$ detector ${ }^{8}$ ) is shown in fig. 4 . The intensity of the predicted spectrum is given in terms of the absolute units indicated in the figure. The normalization for the measured spectrum is arbitrary so only relative comparisons of the spectra are meaningful. The relative shapes of the Compton continua are seen to be in agreement. The discrepancy in the region between the photo peaks is again probably due to scattered radiation.

\section{Results of the detector response calculations}

The program has been used to investigate numerous detector response parameters as a function of the

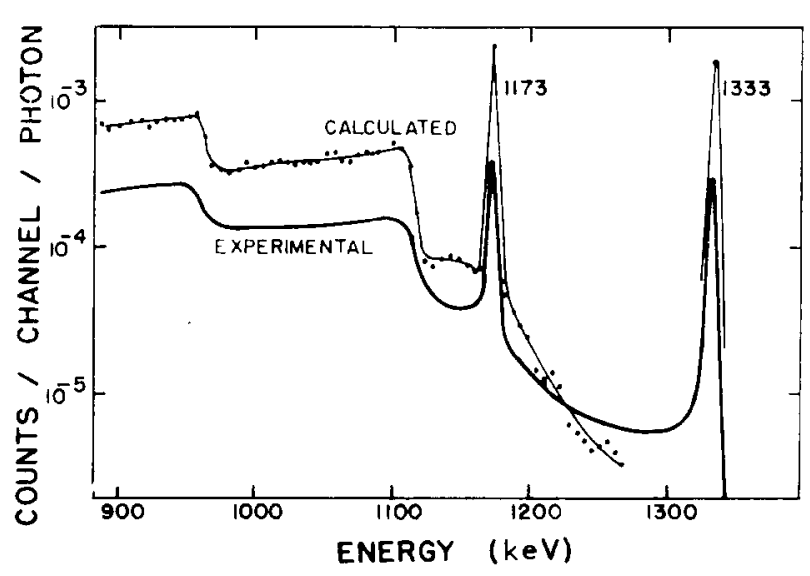

Fig. 4. Comparison of computed and experimental data for the pulse height distribution of ${ }^{60} \mathrm{Co}$ in a $3.5 \mathrm{~mm} \times 2.5 \mathrm{~cm}^{2}$ germanium detector. A channel width of $6 \mathrm{keV}$ was used in the calculation and the results have been normalized to unit incident photon flux. Experimental data by Ewan and Tavendale ${ }^{8}$ ).

photon energy and detector thickness. Among the parameters investigated were:

1. the total absorption probability;

2. the intrinsic efficiency;

3. the peak to total ratio;

4. the double escape pair peak efficiency;

5. the pulse height distribution;

6. the fraction of the total absorptions events due to primary photoelectric reactions;

7. the probability for total absorption of secondary electrons;

8. the average secondary electron energy absorbed in the detector.

The computations were made for photon energies of $0.1364,0.3203,0.6616,1.3325$ and $2.6145 \mathrm{MeV}$ corresponding to gamma radiation from ${ }^{57} \mathrm{Co},{ }^{51} \mathrm{Cr}$, ${ }^{137} \mathrm{Cs},{ }^{60} \mathrm{Co}$ and $\mathrm{ThC}^{\prime \prime}$, respectively. In all cases the source is assumed to be a parallel beam uniformly illuminating and normally incident on the planar detector surface. Fully depleted detectors of germanium and silicon having thicknesses equal to $1,3.5,8$ and $12 \mathrm{~mm}$ were chosen for the calculations. In all cases, the detector is assumed to be a right circular cylinder surrounded by vacuum. The area of the front surface is $2.5 \mathrm{~cm}^{2}$ and there is no dead layer.

The results of the calculations for the total absorption probability, that is, the probability that a photon will interact and lose all its energy in the detector, are shown in figs. 5 and 6 for germanium and silicon detectors respectively. Experimental data obtained by Bertolini et al. ${ }^{9}$ ) for the total absorption probability in a silicon detector are also shown for reference in fig. 6 . The probability for total absorption is much larger in 


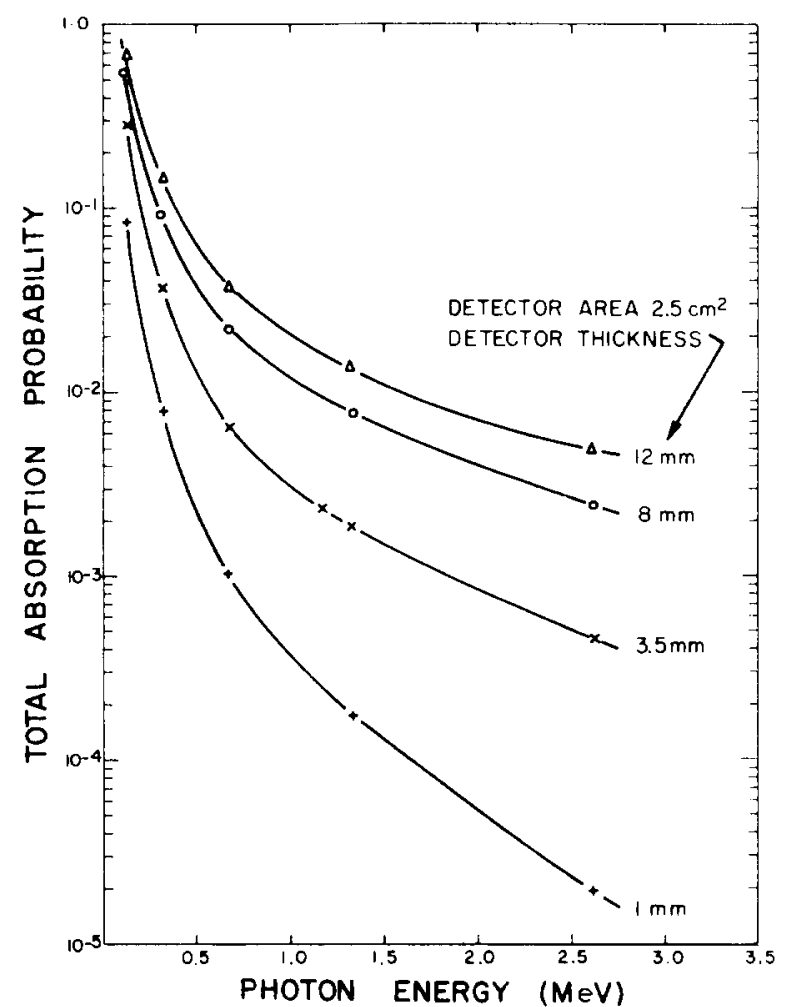

Fig. 5. Total absorption probability for germanium detectors.

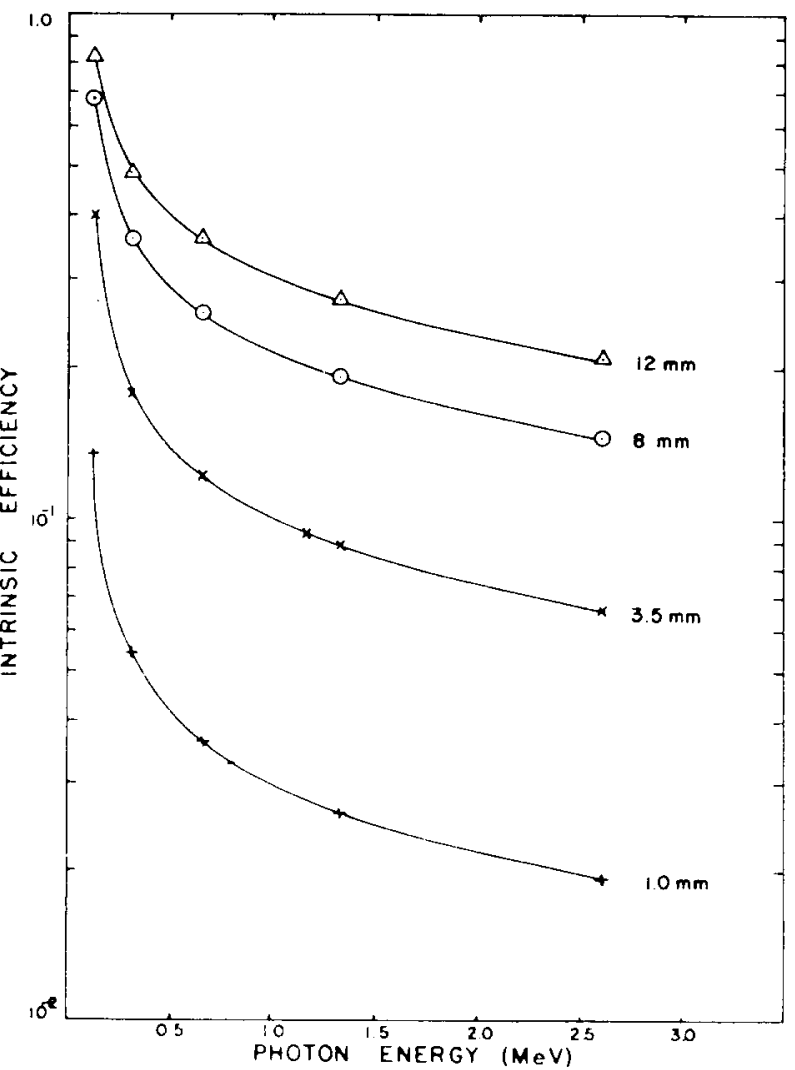

Fig. 7. Intrinsic efficiency of germanium detectors.

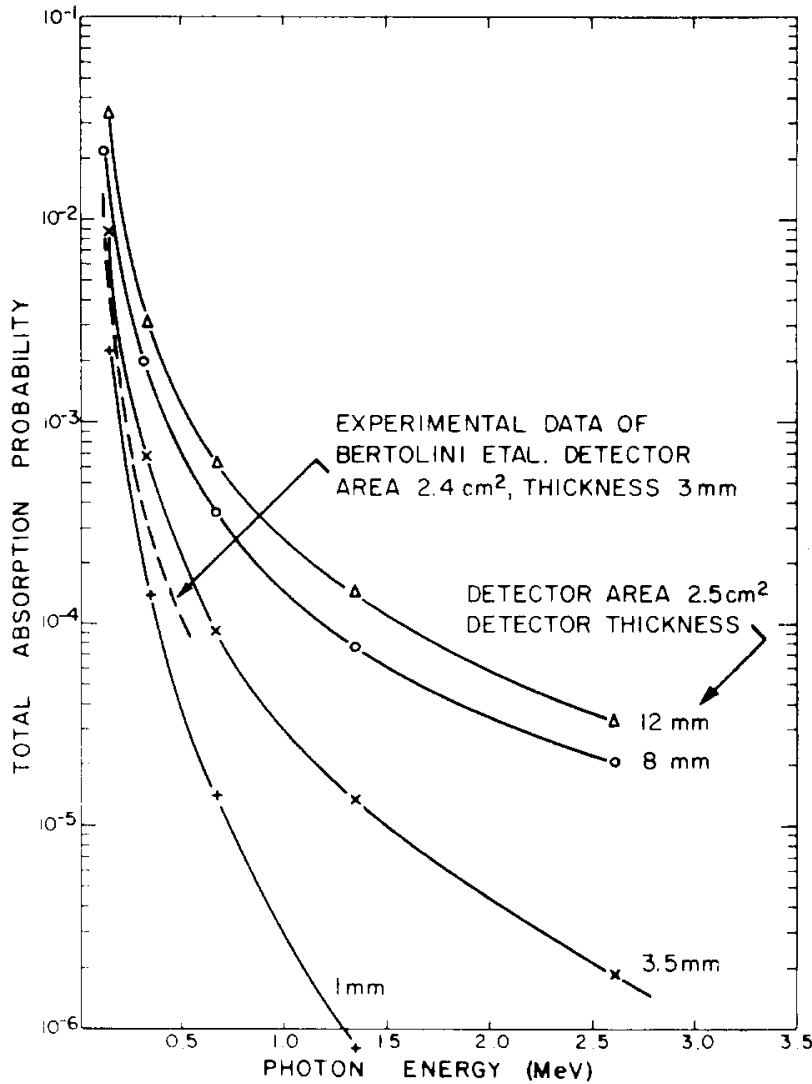

Fig. 6. Total absorption probability for silicon detectors. Dashed curve gives experimental data of Bertolini et al. ${ }^{9}$ ).

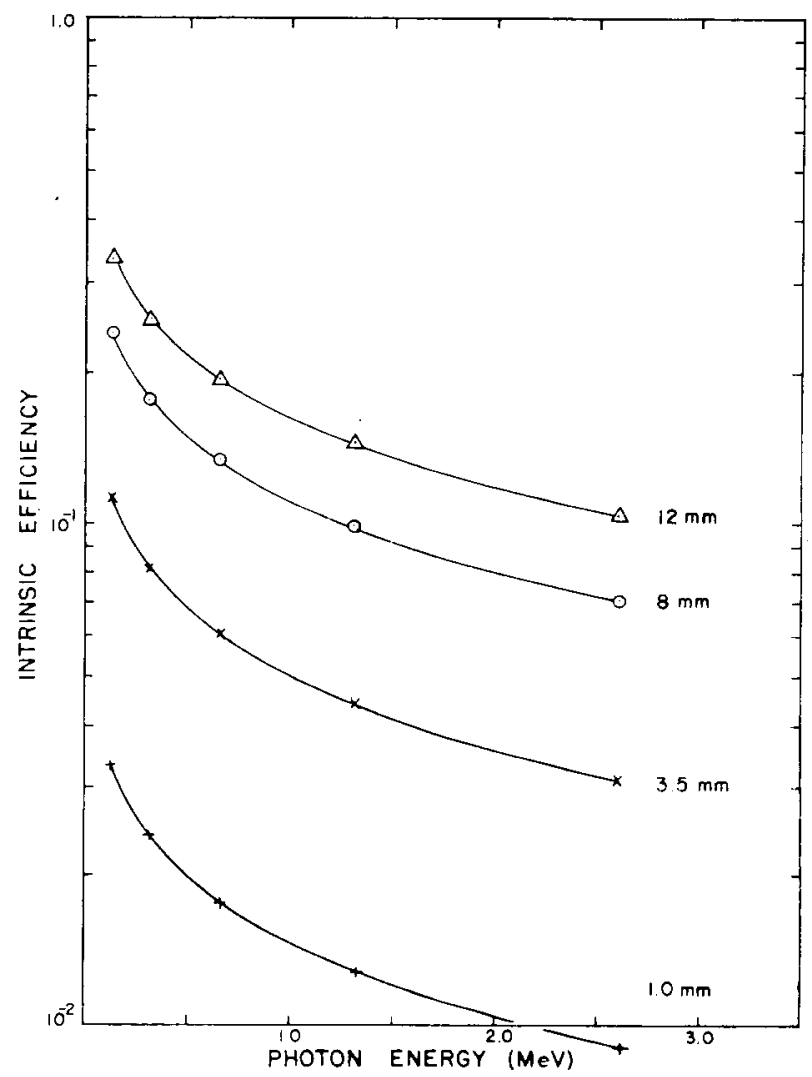

Fig. 8. Intrinsic efficiency of silicon detectors. 


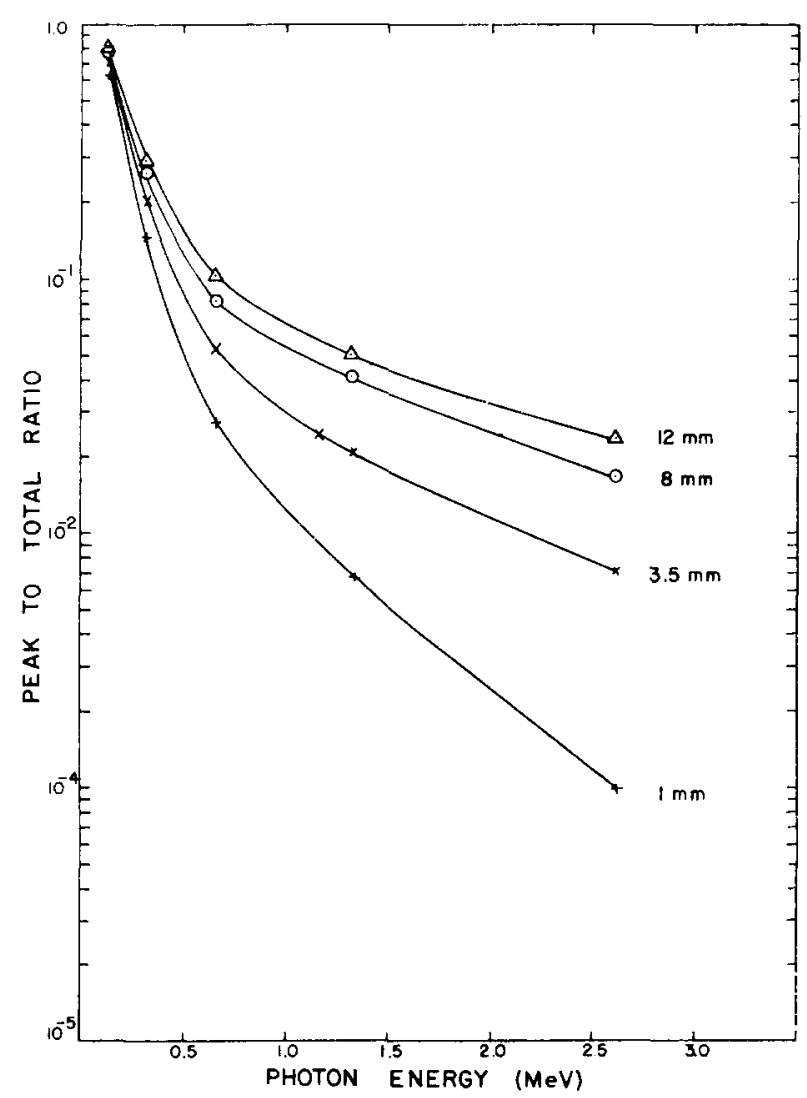

Fig. 9. Peak to total ratio for germanium detectors.

germanium than in silicon due to the larger photoelectric absorption cross section. Because the procedure for calculating electron leakage is inaccurate for electron energies greater than $2 \mathrm{MeV}$, the computed points at $2.614 \mathrm{MeV}$ are probably too high. The error in calculating the leakage will be greater for electrons produced in primary photoelectric reactions than those produced in primary Compton events since the average energy of the photoelectrons is larger. Therefore, the error in the total absorption probability data is expected to decrease with increasing detector thickness because the fraction of total absorption events due to primary photoelectric reactions decreases with detector thickness (figs. 13 and 14).

Results of the calculations for the intrinsic efficiency, i.e., the probability that the incident photon will interact and deposit at least part of its energy in the detector, are presented in figs. 7 and 8 . The differences between the germanium and silicon detectors are less marked than for the total absorption probability data since the intrinsic efficiency is approximately proportional to the total cross section. Therefore at $1 \mathrm{MeV}$ and above, where Compton scattering is the predominant inter-

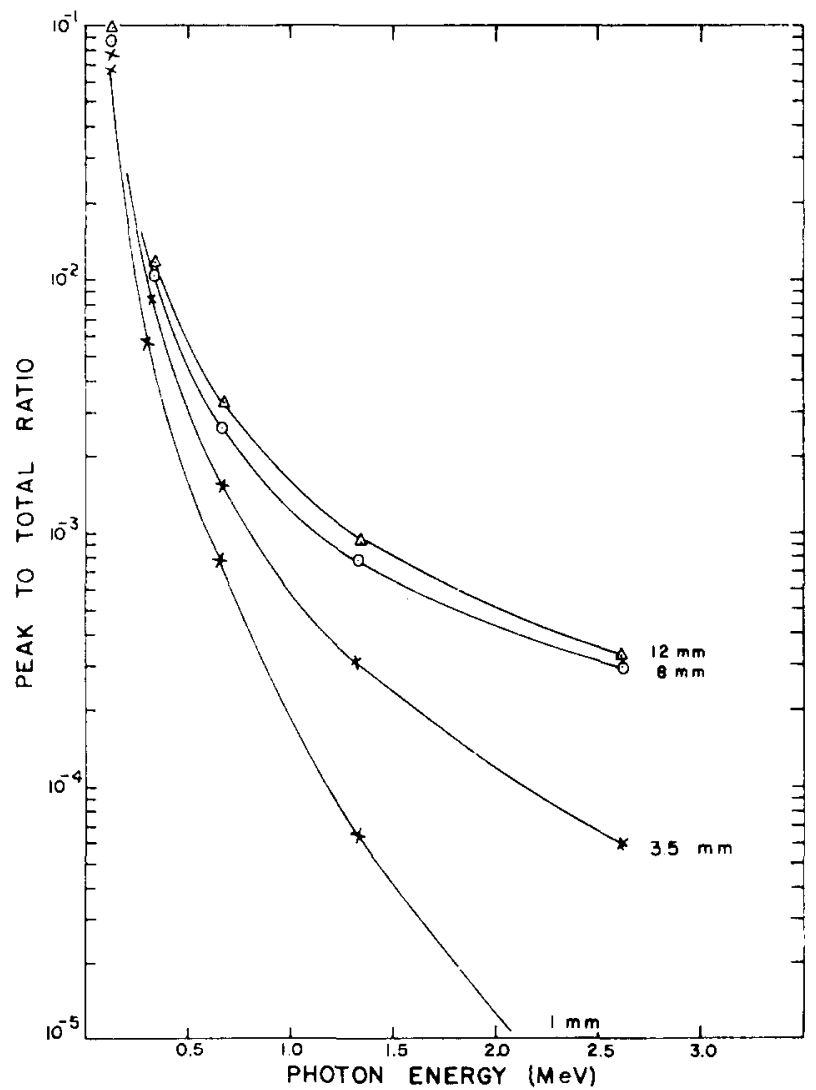

Fig. 10. Peak to total ratio for silicon detectors.

action mode, the intrinsic efficiency in germanium is only a factor of about 2 larger than in silicon reflecting the fact that germanium is approximately twice as dense as silicon. At lower energies, the germanium detectors have a significantly larger intrinsic efficiency because of the larger photoelectric cross section.

Graphs of the peak to total ratio, defined as the ratio of the total absorption probability to the intrinsic efficiency, are shown in figs. 9 and 10 . The peak to total ratio is much larger in germanium detectors because of the greater total absorption probability.

Figs. 11 and 12 show the results of the calculations for the double escape peak efficiency, defined as the probability that the incident photon interacts via the pair production reaction with all the electron and positron energy being absorbed in the detector, while both annihilation quanta escape without interaction. The pair production cross section varies only as $Z^{2}$ rather than $Z^{5}$, as does the photoelectric cross section, so the differences between the double escape peak efficiency in germanium and silicon detectors are not as large as the differences in the total absorption probability. Consequently, silicon detectors compare much 


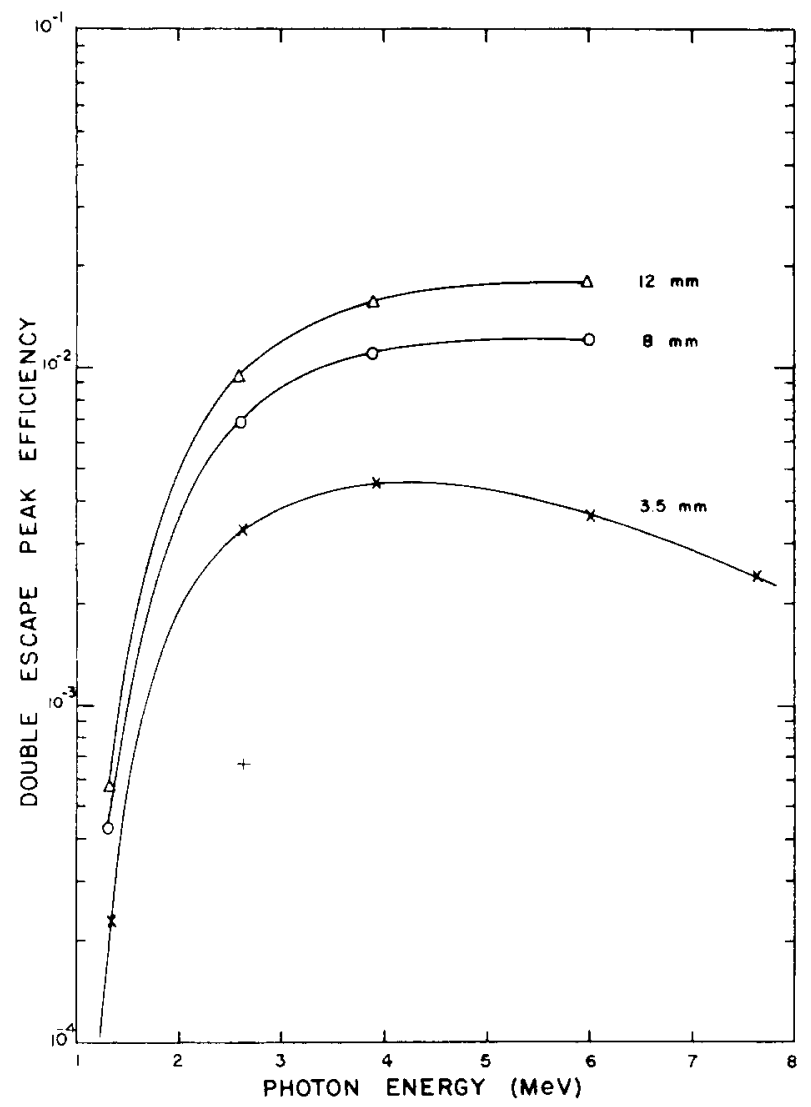

Fig. 11. Double escape peak efficiency of germanium detectors. Only one data point, marked by the $(+)$ at $2.614 \mathrm{MeV}$, was computed for the $1 \mathrm{~mm}$ thick detector.

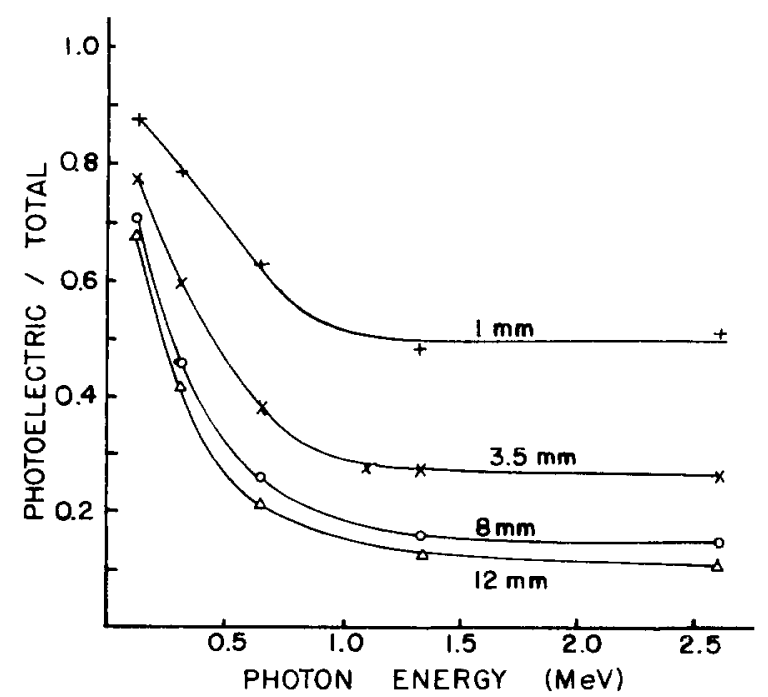

Fig. 13. Fraction of total absorption events in germanium detectors due to primary photoelectric absorption interactions.

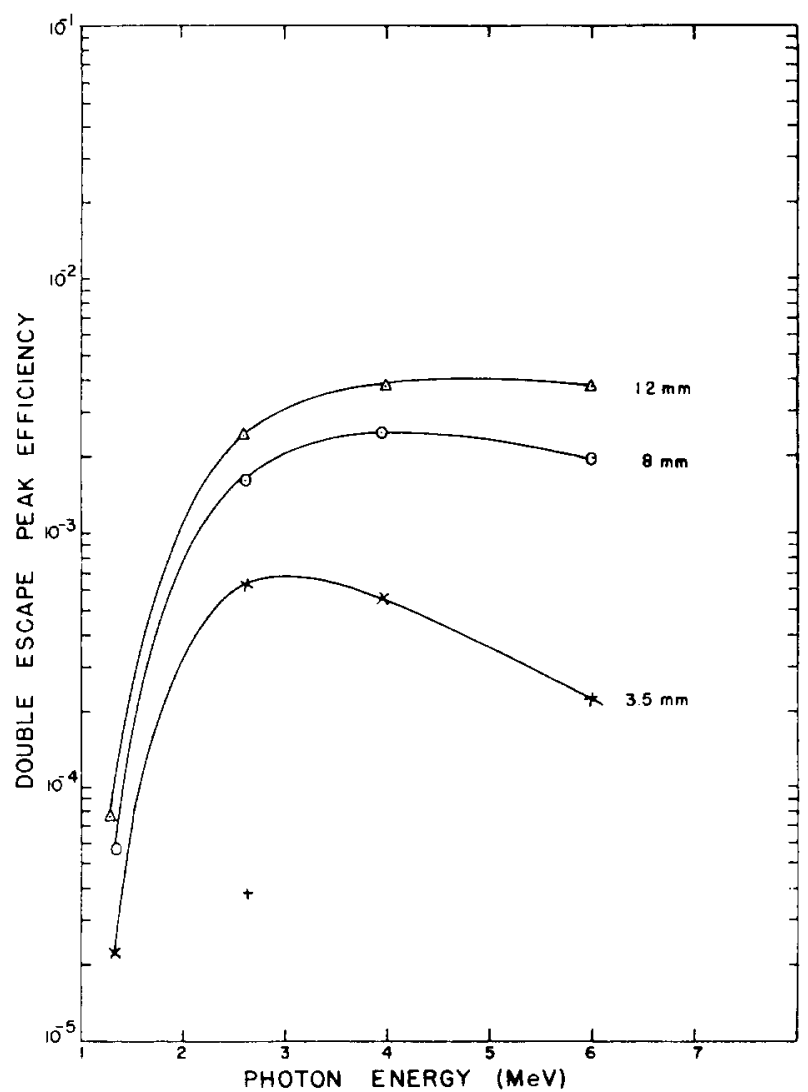

Fig. 12. Double escape peak efficiency of silicon detectors. Only one data point, marked by the (+) at $2.614 \mathrm{MeV}$, was computed for the $1 \mathrm{~mm}$ thick detector.

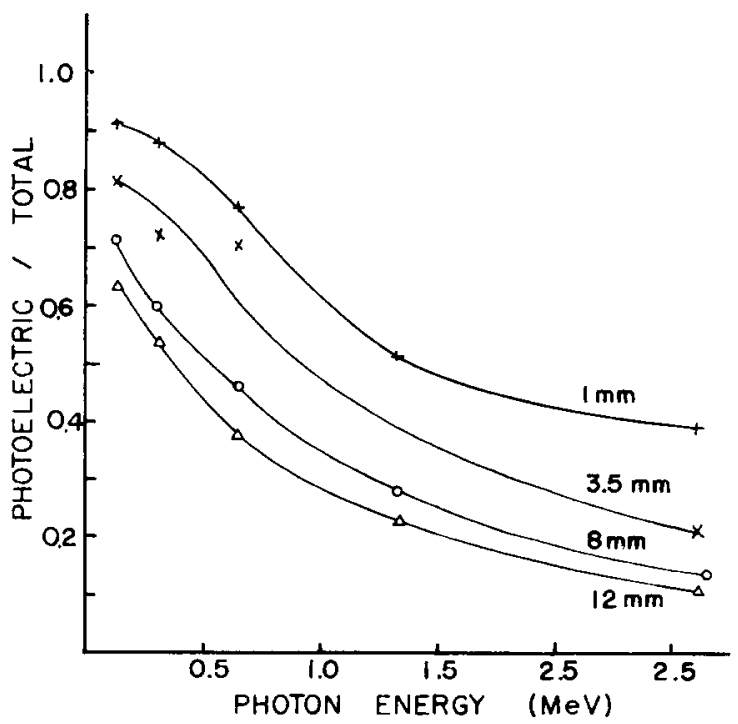

Fig. 14. Fraction of total absorption events in silicon detectors due to primary photoelectric absorption interactions. 


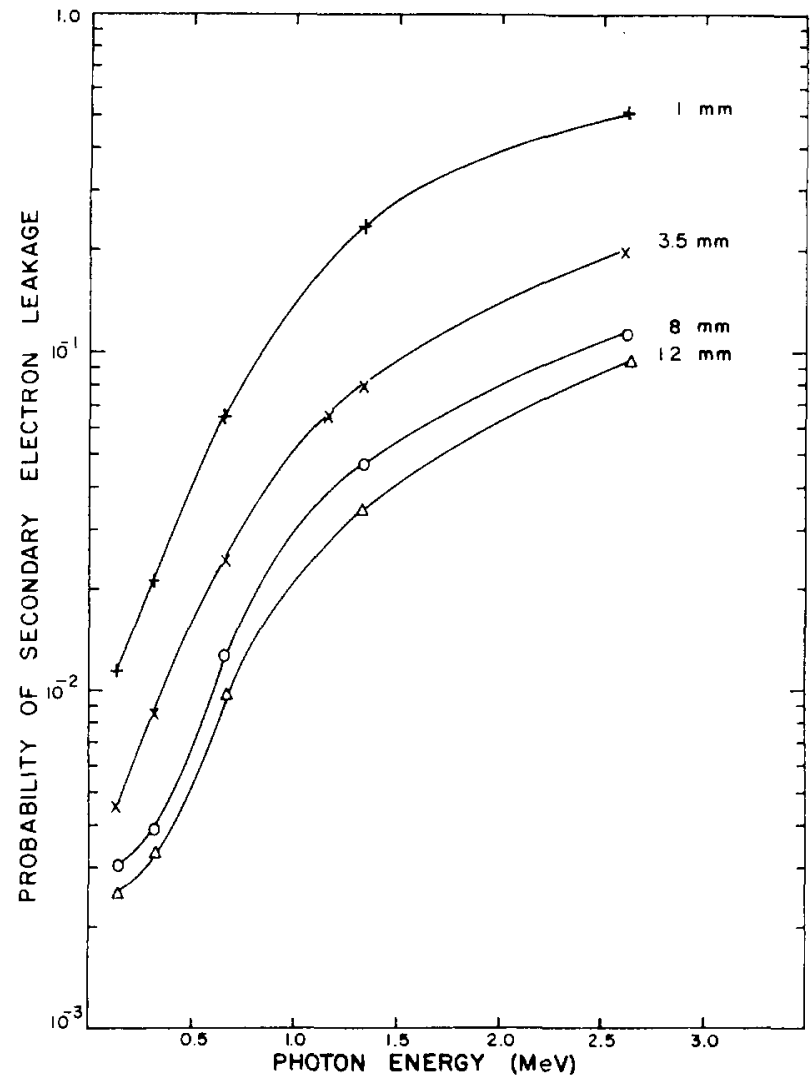

Fig. 15. Probability of secondary electron leakage from germanium detectors.

more favorably with germanium detectors when used as pair spectrometers.

The program has also been used to calculate detector response parameters which are not of direct use in analyzing experimental data but which are useful in understanding the detection processes. For example, the data in figs. 13 and 14 give the fraction of total absorption events that result from primary photoelectric events. It can be seen that in the thick detectors, as much as 90 per cent of the total absorption events can result from primary interactions other than photoelectric absorption.

Given that a secondary electron is produced in the detector, the data in figs. 15 and 16 give the average probability that at least part $(>3 \mathrm{keV})$ of the electron energy will escape from the detector. For pair production events where a secondary electron-positron pair is formed, the energies of the pair were summed and the leakage probability based on the condition that at least part of the total energy escaped from the detector. The leakage probability in silicon detectors is larger than in germanium detectors as expected. The average fraction

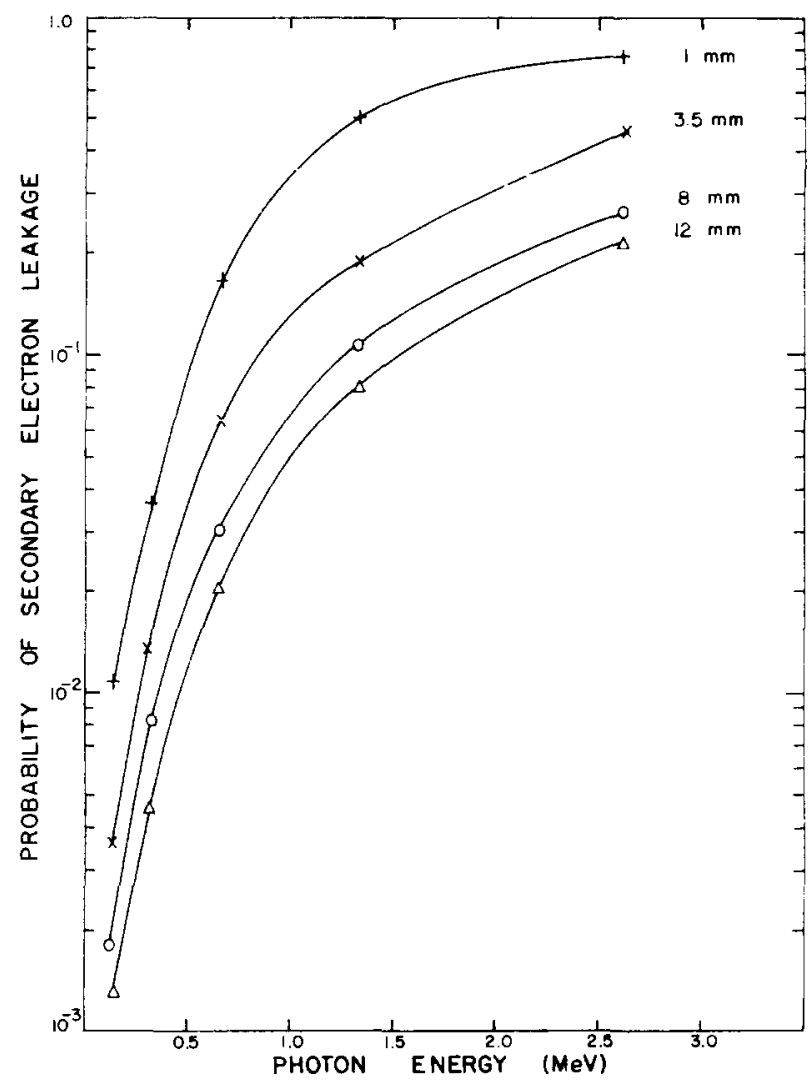

Fig. 16. Probability of secondary electron leakage from silicon detectors.

of the secondary electron energy lost by leakage out of the detector is presented in figs. 17 and 18.The data were obtained by forming the average $\left\langle E_{\mathrm{a}} / E_{0}\right\rangle$ and subtracting the result from unity. $E_{0}$ is the energy of the electron created in a particular Monte Carlo history, $E_{\mathrm{a}}$ is the amount of energy absorbed in the detector, and the average is taken over all the histories. The results obtained in this way do not differ appreciably from the results obtained by using the ratio of the averages; i.e., $\left\langle E_{\mathrm{a}}\right\rangle \mid\left\langle E_{0}\right\rangle$. The average energy of the secondary electron is indicated by the dashed lines in the figures.

The computed pulse height spectra produced by ${ }^{137} \mathrm{Cs}$ gamma rays $(0.6616 \mathrm{MeV})$ for various thicknesses of germanium and silicon detectors are shown in figs. 19 and 20. The absolute intensities of the Compton continua are expressed in units of counts per $\mathrm{keV}$ per incident photon. The data for the photopeaks give the total count under the peak. The intensity of the photopeak can be obtained from the scale at the left of the figure but, in this case, the units are counts per incident photon. The change in shape of the Compton continua with detector thickness is due to the combined effects 


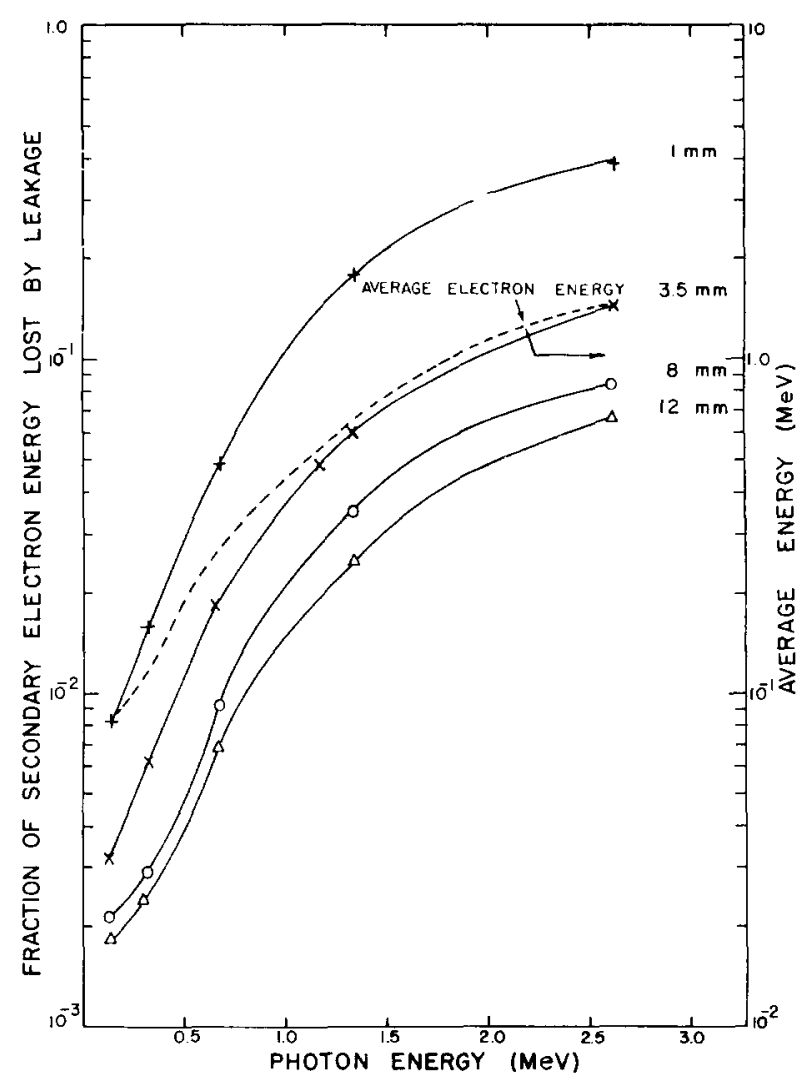

Fig. 17. Average fraction of secondary electron energy lost by leakage from germanium detectors. Dashed curve gives the average energy of the secondary electrons.

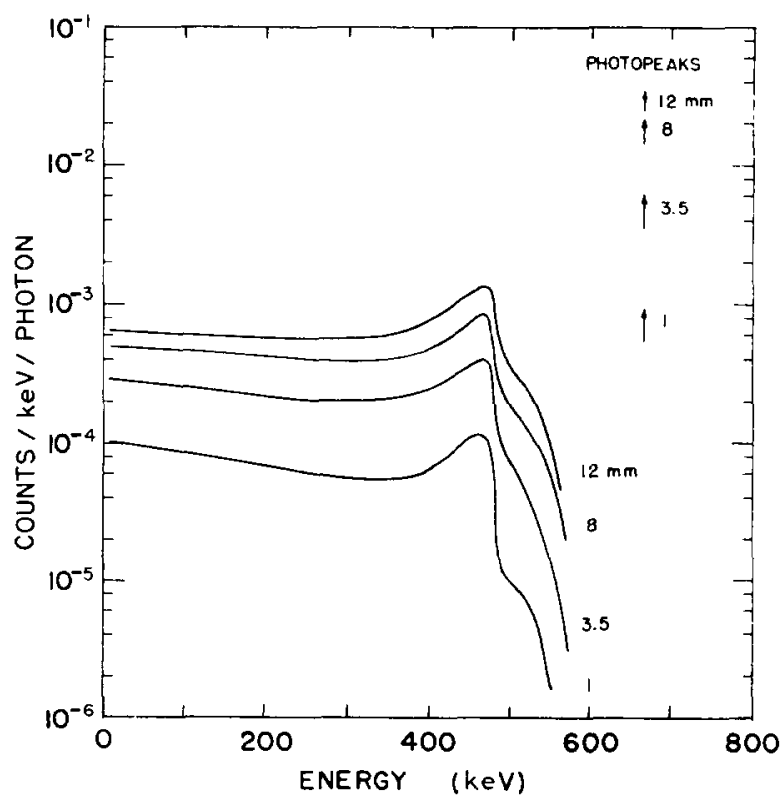

Fig. 19. Pulse height distributions produced by ${ }^{137} \mathrm{Cs}$ photons in germanium detectors. Photopeaks are indicated by the short arrows which give the total number of counts under the photopeak per incident photon.

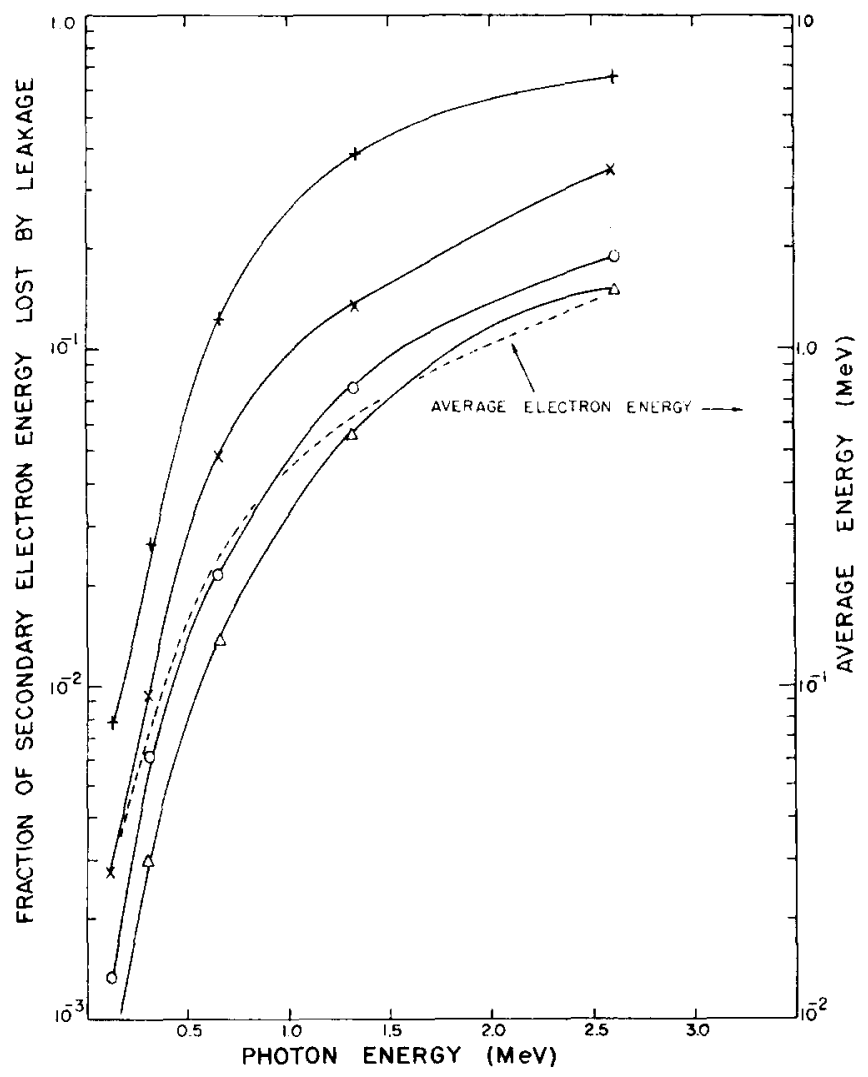

Fig. 18. Average fraction of secondary electron energy lost by leakage from silicon detectors. Dashed curve gives the average energy of the secondary electrons.

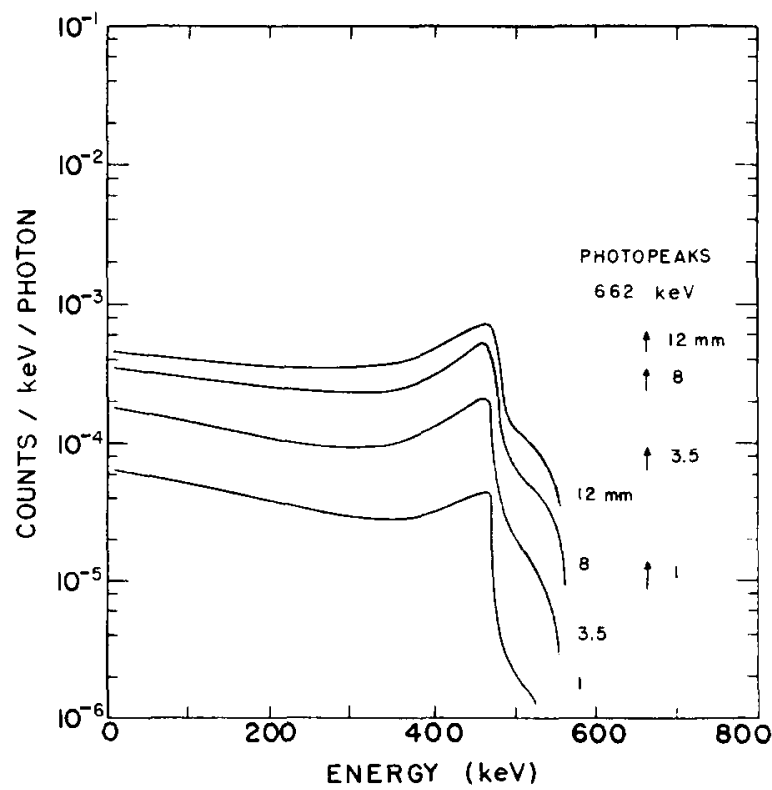

Fig. 20. Pulse height distributions produced by ${ }^{137} \mathrm{Cs}$ photons in silicon detectors. Photopeaks are indicated by the short arrows which give the total number of counts under the photopeak per incident photon. 


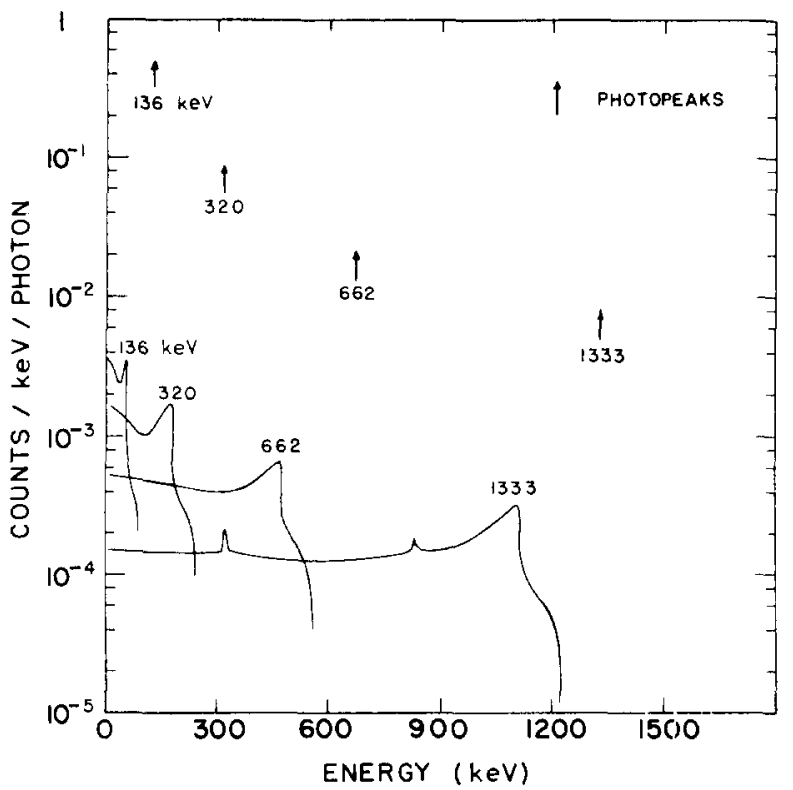

Fig. 21. Pulse height distributions produced by $1333,662,320$ and $136 \mathrm{keV}$ photons in an $8 \mathrm{~mm}$ thick germanium detector. Photopeaks are indicated by the short arrows which give the total number of counts under the photopeak per incident photon.

of electron leakage and multiple photon interactions in the detector. The spectra for germanium and silicon are quite similar in shape and intensity due to the fact that the probabilities for Compton interactions in the two materials are nearly equal. The significant difference between the spectra for the two materials is the intensity of the photopeaks. In germanium, the photopeaks are almost two orders of magnitude more intense than in silicon.

Figs. 21 and 22 show the dependence of pulse height spectra on photon energy for a detector thickness of $8 \mathrm{~mm}$. The absolute intensity of the Compton continua are expressed in units of counts per $\mathrm{keV}$ per incident photon. As above, the data for the photopeaks give the total number of counts under the peak per incident photon. At low energies, there is a pronounced minimum in the Compton continuum. As the photon energy is increased, the relative position of the minimum shifts and it becomes less pronounced. The primary reason for this variation in the shape of the continua is the dependence of the energy distribution for Compton electrons on the incident photon energy. At the highest energies shown, the single and double escape peaks begin to become noticeable.

\section{Conclusions}

Comparison of calculated results with experimental data have shown that the Monte Carlo code provides

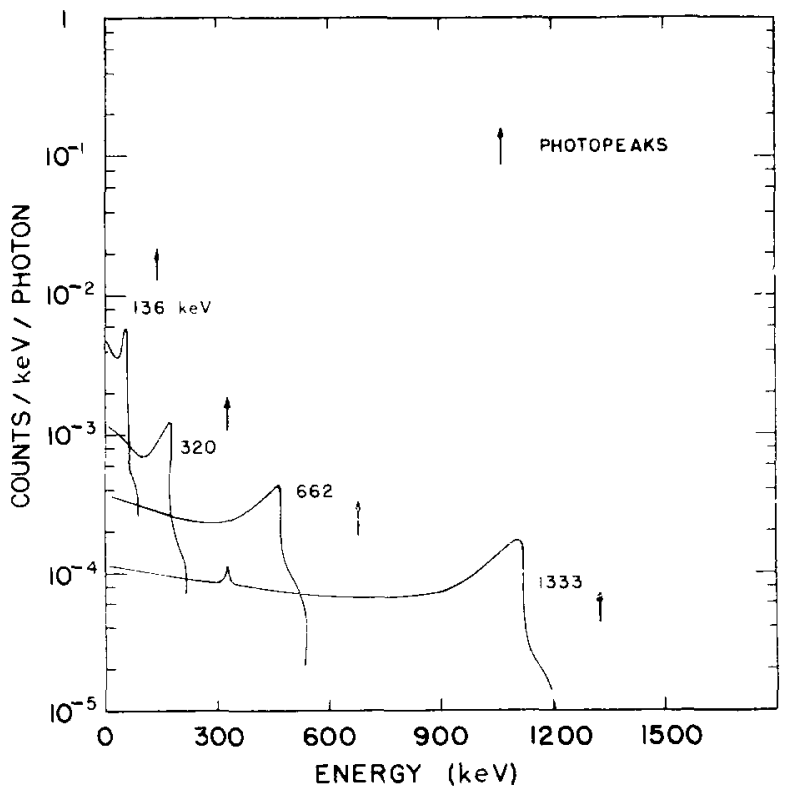

Fig. 22. Pulse height distributions produced by $1333,662,320$ and $136 \mathrm{keV}$ photons in an $8 \mathrm{~mm}$ thick silicon detector. Photopeaks are indicated by the short arrows which give the total number of counts under the photopeak per incident photon.

reasonably accurate predictions of the gamma ray response characteristics of semiconductor detectors. The validity of the calculations is restricted to those cases where the secondary electron energy is less than $2 \mathrm{MeV}$. Since the energy of a photoelectron is approximately equal to the energy of the incident photon but the energies of Compton electrons and pair electrons are considerably less, the restriction imposed on the incident photon energy depends on the type of detector response parameter being investigated. The calculations of total absorption are expected to be valid for incident photon energies up to about $2.5 \mathrm{MeV}$ because most of the total absorption events are due to primary interactions other than photoelectric absorption. Similarly, the calculations of the double escape peak efficiency are expected to be valid for photon energies up to about $6 \mathrm{MeV}$ since the average energy of pair electrons is then $2.5 \mathrm{MeV}$. The limitation on electron energy places no restriction on the validity of the intrinsic efficiency calculations.

The Monte Carlo code developed for these calculations will be made available through the code center at the Argonne National Laboratories. The program is written in the MAD language (Michigan Algorithm Decoder) for an IBM 7090 computer and will require the use of MESS (Michigan Executive System and Subroutines). The amount of computation time required per Monte Carlo history depends to some extent 
on the detector size and incident photon energy but, typically, these times are on the order of $0.01 \mathrm{sec} /$ history for histories beginning with a photoelectric event, $0.03 \mathrm{sec} /$ history for initial Compton events and $0.05 \mathrm{sec} /$ history for initial pair production events. The computations for each detector thickness-photon energy combination reported above required less than 5 min of computer time.

\section{References}

1) E. Storm, E. Gilbert and H. Israel, LA-2237, Los Alamos Scientific Laboratory.
2) C. M. Davisson and R. D. Evans, Rev. Mod. Phys. 24 (1952) 79.

3) H. Kahn, RM-1237, the Rand Corporation.

4) W. Heitler, The quantum theory of radiation, $3^{\mathrm{d}}$ ed. (Oxford U. P., 1954) p. 259.

5) K. M. Wainio and G. F. Knoll, Bull. Am. Phys. Soc. 10, no. 6 (1965) 719.

6) A. T. Nelms, U. S. NBS Circular 577.

7) C. D. Zerby and H. S. Moran, ORNL-2454, Oak Ridge National Laboratory.

8) G. T. Ewan and A. J. Tavendale, Can. J. Phys. 42 (1964) 2286.

9) G. Bertolini, F. Cappellani and G. Restelli, Nucl. Instr. and Meth. 32 (1965) 86. 